\title{
Evidence of Quorum Sensing in Cyanobacteria by Homoserine Lactones: The Origin of Blooms
}

\author{
Natalia Herrera * and Fernando Echeverri *(D) \\ Group of Organic Chemistry Natural Products, Institute of Chemistry, Universidad de Antioquia, \\ Medellin 050010, Colombia \\ * Correspondence: fernando.echeverri@udea.edu.co (N.H.); natalia.herrera@udea.edu.co (F.E.); Tel.: \\ $+57-300-318-8876$ (N.H.)
}

check for updates

Citation: Herrera, N.; Echeverri, F. Evidence of Quorum Sensing in Cyanobacteria by Homoserine Lactones: The Origin of Blooms. Water 2021, 13, 1831. https:// doi.org/10.3390/w13131831

Academic Editors: Naresh Singhal and Helvi Heinonen-Tanski

Received: 28 April 2021

Accepted: 25 June 2021

Published: 30 June 2021

Publisher's Note: MDPI stays neutral with regard to jurisdictional claims in published maps and institutional affiliations.

Copyright: (c) 2021 by the authors. Licensee MDPI, Basel, Switzerland. This article is an open access article distributed under the terms and conditions of the Creative Commons Attribution (CC BY) license (https:// creativecommons.org/licenses/by/ $4.0 /)$.

\begin{abstract}
Although several theories have been postulated to explain cyanobacterial blooms, their biochemical origin has not yet been found. In this work, we explore the existence of bacterial communication, called quorum sensing, in Microcystis aeruginosa and Cylindrospermopsis raciborskii. Thus, the application of several known acylhomoserine lactones to cultures of both cyanobacteria causes profound metabolic. At $72 \mathrm{~h}$ post-application, some of them produced substantial increases in cell proliferation, while others were inhibitors. There was a correlation with colony-forming activity for most of them. According to ELISA analysis, the microcystin levels were increased with some lactones. However, there was a clear difference between M. aeruginosa and C. raciborskii culture since, in the first one, there was an inducing effect on cell proliferation, while in C. raciborskii, the effects were minor. Besides, there were compound inhibitors and inducers of microcystins production in M. aeruginosa, but almost all compounds were only inducers of saxitoxin production in C. raciborskii. Moreover, each lactone appears to be involved in a specific quorum sensing process. From these results, the formation of cyanobacterial blooms in dams and reservoirs could be explained since lactones may come from cyanobacteria and other sources as bacterial microflora-associated or exogenous compounds structurally unrelated to lactones, such as drugs, industrial effluents, and agrochemicals.
\end{abstract}

Keywords: cyanobacteria; Microcystis aeruginosa; Cylindridrospermopsis raciborskii; blooms; proliferation; toxin; Quorum Sensing; autoinducers lactones

\section{Introduction}

The increase of cyanobacterial blooms has been evident in recent years worldwide, in water bodies dedicated to obtaining potable water, energy generation, fishing, and some sports [1]. These blooms are a sudden and excessive proliferation of cyanobacteria, usually with the synthesis of toxins. Their formation has been attributed to increased nutrients ( $N$ and $\mathrm{P}$ ) and high solar radiation temperatures. However, there is no total certainty about their exact origin [2].

Exposure to water-containing cyanobacterial blooms is a risk to animal and human health due to toxins that mainly affect the liver and kidneys, among other organs $[3,4]$. Similarly, fish farming is an industry threatened in terms of its survival and operation [5] since there is proof of the accumulation of cyanotoxins in fish through the trophic chain [6].

Although there is no conclusive evidence of chemical communication in cyanobacteria, particularly quorum sensing, blooms' metabolic development might occur through the same process as in bacteria, including sudden cell proliferation, colony formation, production of a biofilm, and synthesis of toxins. Because cyanobacteria are closely related to Gram (-) bacteria [7], we believe that in both cases, the same type of autoinducer molecules are involved, specifically lactones with side chains of different lengths. Therefore, in this paper, we analyzed the effects of several homoserine lactones on the proliferation and 
development of Microcystis aeruginosa and Cylindrospermopsis raciborskii to establish the functioning of quorum sensing in these cyanobacteria.

So, it is probable that the formation of exogenous molecules structurally or functionally like natural lactones is triggered by human and industrial activity in water bodies. Understanding the route through which blooms are elicited and the responsible agents could generate new viewpoints and alternatives for their control and prevention. The use of physical and chemical methods is a colossal task due to the enormous volumes of water that would need to be treated and the costs involved. Therefore, preventing these substances from reaching water bodies could be a more feasible and effective alternative to preventing the development of blooms.

\section{Experimental}

\subsection{Microcystis aeruginosa and Cylindrospermopsis raciborskii Cultures}

Cultures were made on BG11 and WC medium [8]. For Microcystis aeruginosa, $10 \mathrm{~mL}$ of a cyanobacterial bloom sample from Riogrande II dam (Antioquia, Colombia) in June 2015 was obtained and transferred to the BG11 medium. The culture was maintained in a $20 \mathrm{~h}: 4 \mathrm{~h}$ light/darkness photoperiod at $25{ }^{\circ} \mathrm{C}$, with illumination at $150 \mu \mathrm{mol}$ photons $\mathrm{m}^{-2} \mathrm{~s}^{-1}$. under constant aeration using a Desum AC 9904 air pump. After seven days of cultivation, $100 \mathrm{~mL}$ was taken and centrifuged at $5000 \mathrm{rpm}$ for $5 \mathrm{~min}$ in a Hermnle Z $326 \mathrm{~K}$ centrifuge (HERMLE Labortechnik GmbH Wehingen Germany). The supernatant was transferred to $300 \mathrm{~mL}$ of BG11 medium (UTEX, Austin USA) for seven days. Then, three new cultures were made every five days, taking $10 \mathrm{~mL}$ of the culture and another $300 \mathrm{~mL}$ of BG11 medium. Regular microscopic inspection revealed that biomass of heterotrophic bacteria remained well under $1 \%$ of the total biovolume. Taxonomic identification at the intragenic level was based on specific literature for each group of cyanobacteria [9].

Cylindrospermopsis raciborskii was isolated from Pedra Branca State Park, Río de Janeiro (Brazil) in 2012 and 2013 and identified in the Culture Collection of the Laboratory of Ecology and Physiology of Phytoplankton, University of Rio de Janeiro State (UERJ). Cultures were not grown axenically and the biomass of heterotrophic bacteria remained under $1 \%$ of total volume.

For the maintenance of the cultures, M. aeruginosa was cultivated in BG11 medium with continuous stirring, and this medium was changed every four days. For $C$. raciborskii an equal mixture of WC and BG11 medium was used and changed every fortnight but daily manually stirring.

All assays were undertaken in triplicate.

\subsection{Cell Counts}

For the cell counts, $200 \mu \mathrm{L}$ each of cyanobacteria strains were taken and then diluted in distilled water according to each concentration. The counts were made using Neubauer's chamber coupled to an optic microscope (Olympus, Tokyo, Japan). For the calculation, the number of cells per milliliter was multiplied by the chamber's dilution factor.

The number of colonies per milliliter was also found to determine the direct effect of substances on colony formation.

\subsection{Inducer Lactones}

Vanillin and lactones were purchased in SIGMA-ALDRICH (St Louis, MO, USA); they were dissolved in methanol to obtain a stock of $1 \mathrm{mg} / 10 \mathrm{~mL}$ concentration. From that, aqueous solutions for the assay were prepared by dilution with sterile water.

These lactones corresponded to the compounds most commonly reported to be involved in QS in Gram (-) bacteria [10]: N-(3-oxodecanoyl-L-homoserine lactone (A) (3-oxoC10 AHL), N-(3-oxododecanoyl)-L-homoserine lactone) (B) (3-oxo-C10 AHL), N-hexanoylL-homoserine lactone (C) (C6 AHL), N-decanoyl-DL-homoserine (C10 AHL), N-decanoylhomoserinelactone (D) (C10 AHL), N-heptanoyl-L-homoserine lactone (E) (C7 AHL), Ndodecanoyl-L-homoserine lactone (F) (C12 AHL), N-octanoyl-L-homoserine lactone (G) (C8 
AHL), N-(3-oxooctanoyl)-L- homoserine lactone (H) (3-oxo-C8 AHL), and N-butyryl-DLhomoserine lactone (I) (C4 AHL).

\subsection{Effect of Substances}

\subsubsection{Effect of Lactones on Cell Proliferation and Colony Formation of M. aeruginosa}

$M$. aeruginosa growth induction assays were conducted under the same growing conditions. Initially, $20 \mathrm{~mL}$ of stock culture (approximately $8.15 \times 10^{5}$ cells $/ \mathrm{mL}$ ) was mixed with $200 \mathrm{~mL}$ of BG11 medium. The cultures were exposed for $72 \mathrm{~h}$ to each lactone at a concentration of $4 \mathrm{nM}$. A growth control experiment under the same conditions but without lactone was employed. After that, samples were taken for spectrophotometric readings, cell counts, and microscope observations at $0 \mathrm{~h}$ and $72 \mathrm{~h}$. Through the following equation, the growth induction percentage was calculated:

$\%$ of Cyanobacteria Growth Induction $=(($ Initial Treatment - Final Treatment $) \times 100 /$ Control $)$

where treatment and control were the optical densities of M. aeruginosa exposed and not exposed to the lactones. Finally, the samples were frozen at $4{ }^{\circ} \mathrm{C}$ and stored until ELISA microcystin detection assay.

Approximately $8.15 \times 10^{5}$ cells $/ \mathrm{mL}$ of $M$. aeruginosa were taken and exposed to the nine lactones to determine the induction of colony formation. Colony counts were performed under an optical microscope at days 1, 3, and 6 of the assays. Only colonies with more than five cells were counted.

Inoculums of M. aeruginosa with identical amounts of cells $/ \mathrm{mL}$ were taken and exposed to lactones as above to establish colony formation induction. The number of colonies per milliliter was calculated using Neubauer's chamber coupled to an optic microscope (Olympus, Tokyo, Japan). Only colonies with more than five cells were counted.

\subsubsection{Effect of Vanillin on Induction of M. aeruginosa Growth with Four Lactones}

In a previous work [10], related to the search for inhibitors of cyanobacteria blooms, we found that vanillin was a potent inhibitor of cell proliferation. Because of that, an assay was performed to establish whether the lactones could reverse this effect. Thus, cell and colony counts were performed at 0,48 , and $96 \mathrm{~h}$. The assays were carried out using four lactones, chosen based on induction of cell growth of $M$. aeruginosa according to previous results: $\mathrm{N}$-(3-oxododecanoyl)-L-homoserine lactone (B), $\mathrm{N}$-hexanoyl-L homoserine lactone (C), N-dodecanoyl-L-homoserine lactone (F), N-(3-oxooctanoyl)-L-homoserine lactone (H), which were evaluated at a concentration of $4 \mathrm{nM}$. Additionally, vanillin was also assayed since it previously showed inhibition activity in cyanobacteria [11] at $75 \mathrm{ppm}(328.62 \mathrm{nM})$. Moreover, culture was used without any lactone as a control.

M. aeruginosa cultures $\left(8.15 \times 10^{5}\right.$ cells $\left./ \mathrm{mL}\right)$ were prepared in BG-11 medium for $96 \mathrm{~h}$ with each of the lactones $\mathrm{C}, \mathrm{F}$, and $\mathrm{H}$. Then, vanillin or a mixture of vanillin plus lactone were added. The final concentrations were $4 \mathrm{nM}$ of lactone and $328.62 \mathrm{nM}$ of vanillin. The assays were conducted in triplicate, counting the number of cells and colonies at 0,48 , and $96 \mathrm{~h}$ under the microscope. A control experiment was also carried out using M. aeruginosa cultures without compounds.

Lastly, the cells and colonies' morphology exposed to the three pure lactones and those mixed with vanillin were observed under the microscope.

\subsubsection{Effect of Lactones on Cell Proliferation of Cylindrospermopsis raciborskii}

The growth induction assays of $C$. raciborskii were conducted under the same conditions mentioned before. These were exposed for ten days to each of the lactones at a concentration of $4 \mathrm{nM}$, using a growth control experiment under the same conditions but without lactone. From a stock culture, we took $20 \mathrm{~mL}$, completed to $200 \mathrm{~mL}$ with a culture medium. Then, $300 \mu \mathrm{L}$ of the solution was taken to establish the optical density and the growth induction percentage, which were calculated in the same way as for M. aeruginosa. 


\subsection{Spectrometric Readings}

Optical densities were measured using a Multiskan Spectrum (Thermo Scientific, Waltham, MA, USA) at 450, 570, and 630 nanometers. Each measurement was performed in triplicate for each sample, taking $300 \mu \mathrm{L}$ of each culture.

\subsection{M. aeruginosa Cell and Colony Morphology}

Each of the cultures exposed to the different lactones was observed under an optical microscope to identify growth and morphology, specifically the mucilage formation using acid staining with Chinese ink [12].

\subsection{Analysis of Microcystins by ELISA}

Microcystins were detected by ELISA using the QuantiPlate Kit for Microcystins (Envirologix, Portland, OR, USA), which has a detection limit of 0.16 to $2.5 \mathrm{ppb}$. Optical density (OD) was measured using a microplate reader (ELx $800 \mathrm{NB}$, Bio-Tec Instruments Inc., Winooski, VT, USA). Four controls (negative, calibrator 1: $0.16 \mathrm{ppb}$, calibrator 2: $0.6 \mathrm{ppb}$, and 3:2.5 ppb) were used in each strip of eight wells. The calibration curve was constructed from the microcystin concentration of the kit calibrators and the Bo percentage (OD of each calibrator/OD of negative control $\times 100$ ). Thus, the curve equation was applied to obtain the concentrations samples. For the high-performance liquid chromatography/mass spectrometry (HPLC/MS) analyses, a UHR-QTOF (Impact II- Bruker Germany) was used. The samples were separated using a Chromolith HighResolution C18 column $(50 \times 4.6 \mathrm{~mm})$, kept at $40{ }^{\circ} \mathrm{C}$. The mobile phase consisted of Milli-Q water plus $0.1 \%$ formic acid (A) and acetonitrile plus $0.1 \%$ formic acid (B). Separation was performed using a gradient that increased from $5 \%$ B to $10 \%$ B for $1 \mathrm{~min}$, followed by $50 \%$ (B) by $4 \mathrm{~min}$, to $95 \%$ (B) at $6 \mathrm{~min}$, and at $8 \mathrm{~min}$, returned to the initial conditions. A tuning mix solution was used as a calibrator. The data were analyzed using the Bruker Compass Data Analysis 4.3 (Bruker Daltonics) program.

\subsection{Analysis of Saxitoxin by ELISA}

Saxitoxin (PSP) ELISA (Product No. 52255B), microtiter plate kits were purchased from Abraxis (Eurofins) and assayed according to the manufacturer's instructions.

\subsection{Data Analysis}

To establish statistically significant differences between the cell density measurements obtained in the tests with the different lactones, an ANOVA was applied with a significance level of 0.05 . In cases where statistically significant differences were found, the Tukey test was performed to establish differences in sensitivity between substances. A Spearman correlation analysis was applied with a significance level of 0.05 , using the R Core Team statistical package [13] to analyze correlations between microcystin concentrations concerning lactones.

Comparison between groups was performed with one-way ANOVA followed by Tukey's multiple comparison. $p<0.05$ was considered as significant. ${ }^{*}, p<0.05$; ${ }^{* *}, p<0.01$; $* * *, p<0.001$.

\section{Results}

To demonstrate the occurrence of quorum sensing in M. aeruginosa and Cylindrospermopsis raciborskii, we selected a wide range of lactones (Figure 1). Compounds D and I were analyzed like an enantiomeric mixture. 


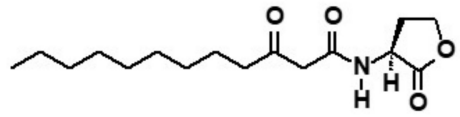

A

N-(3-oxo-dodecanoyl) L-homoserinelactone

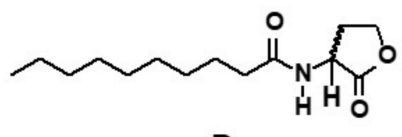

D

N-decanoyl

DL-homoserinelactone

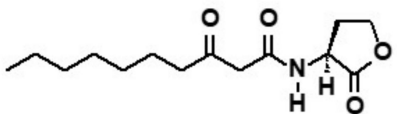

B

N-(3-oxodecanoyl)

L-homoserinelactone

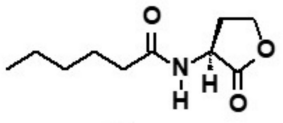

C

N-hexanoyl

L-homoserinelactone<smiles>CCCCCCCC(=O)NC1CCCC1=O</smiles>

G

N-octanoy

L-homoserinelactone

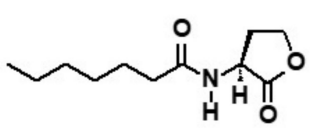

E

N-heptanoyl L-homoserinelactone

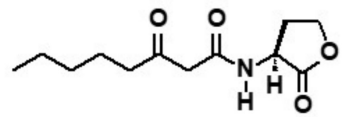

$\mathrm{H}$

N-(3-oxooctanoyl) L-homoserinelactone

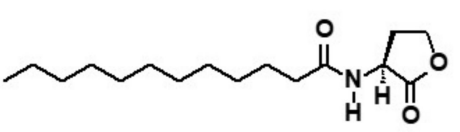

$F$

$\mathrm{N}$-dodecanoyl L-homoserinelactone

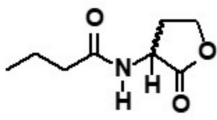

I

N-butiryl-

DL-homoserinelactone

Figure 1. Structure of homoserine lactones assayed against M. aeruginosa and C. raciborskii.

\subsection{Effect of Lactones on Cell Proliferation of Microcystis aeruginosa}

The results of proliferation induction after $M$. aeruginosa exposure to the nine lactones are shown in Figure 2. According to the Tukey test, the main inducers of cell proliferation were N-dodecanoyl (F), N-heptanoyl (E), N-(3-oxooctanoyl) (H), and N-hexanoyl-Lhomoserine lactone (C), which exhibited substantial increments of $178 \%, 168 \%$, and $138 \%$, respectively (Figure 2). These results were significantly different from the control and to N3-oxodecanoyl (A), N-butyryl (I), N-(3-oxododecanoyl) (B), and N-decanoyl-L-homoserine lactone (D), since these compounds showed proliferation percentages below the control of $90 \%, 78 \%, 71 \%, 58 \%$, and $57 \%$, respectively, acting as potent inhibitors in the case of the last four compounds.

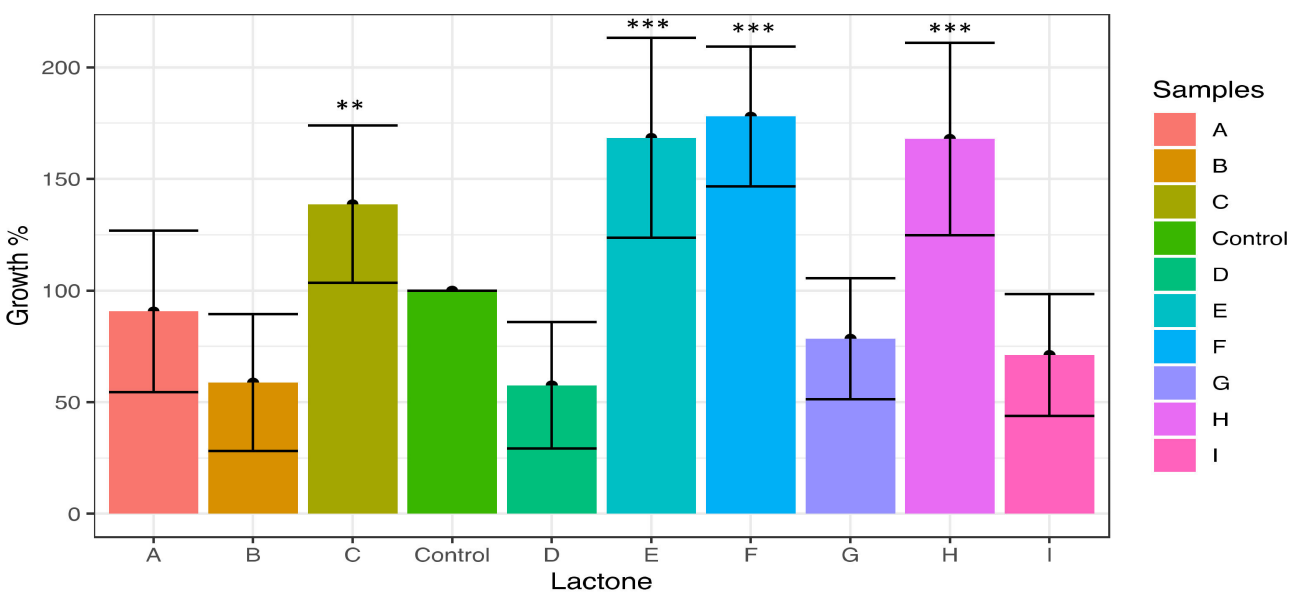

Figure 2. Effects of lactones in cell proliferation of M. aeruginosa with nine homoserine lactones, N3-oxodecanoyl (A), N-(3-oxododecanoyl) (B), N-hexanoyl (C), N-decanoyl (D), N-heptanoyl (E), N-dodecanoyl (F), N-octanoyl (G), N-(3-oxooctanoyl) (H), and N-butyryl-L-homoserine lactone (I) after $72 \mathrm{~h}$ of exposure. The red line indicates the level of proliferation in the control experiment. Powerful inducers of cell proliferation were lactones $\mathrm{E}, \mathrm{F}$, and $\mathrm{H}$ that induce a proliferation close to $75 \%$ of the control, while lactones $\mathrm{B}$ and $\mathrm{D}$ reduce this proliferation by almost $50 \%$ exposure. Whiskers are standard deviations. ${ }^{* *}, p<0.01{ }^{* * *}, p<0.001$. 


\subsection{Effect of Lactones on the Induction of Colony Formation of Microcystis aeruginosa}

One group of lactones promoted colony formation in M. aeruginosa, while a second group did not affect colony formation relative to the control. In the colony-inducing group, the increase in colony formation was $400 \%$ higher than in control. According to the Tukey test, there were two types of statistically different responses on the 6th day of the experiment. The first group (Figure 3) was formed by lactones that are potent inducers of the formation of colonies of $M$. aeruginosa, specifically octanoyl-L-homoserine lactone (G) with an induction percentage of $445 \%$, butyryl (I) at 431\%, N-(3-oxooctanoyl) (H) at $400 \%$, and N-hexanoyl (C) at 395\%, as well as heptanoyl (E) at 313\% and N-dodecanoyl-Lhomoserine lactone $(\mathrm{F})$ at $395 \%$.

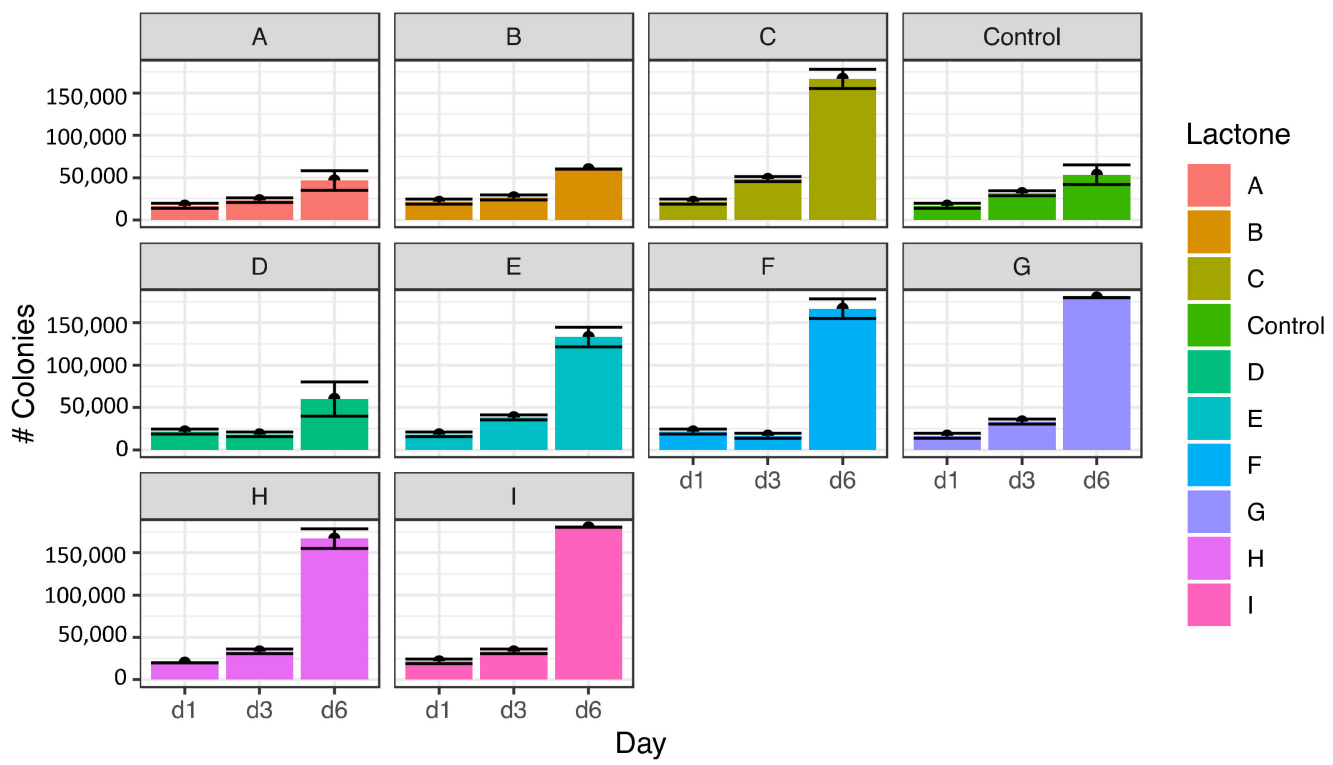

Figure 3. Induction of the formation of Microcystis aeruginosa colonies exposed to the nine lactones for six days. Compounds: N-(3-oxodecanoyl) (A), N-(3-oxododecanoyl) (B), N-hexanoyl (C), decanoyl (D), heptanoyl (E), N-dodecanoyl (F), N-octanoyl (G), N-(3-oxooctanoyl) (H), N-butyryl (I). Lactones C, F, G, H, and I, and to a lesser degree E, were potent colony inducer compounds with values close to $400 \%$ or more, but lactones A, B and were practically inactive. Whiskers are standard deviations, and bars are mean values among replicates.

In the second group, lactones N-3-oxodecanoyl (A), N-(3-oxododecanoyl) (B), and $\mathrm{N}$-decanoyl-L-homoserine lactone (D) were practically inactive like colonies inducers of M. aeruginosa (Figure 3).

\subsection{Effect of Lactones on Colony Morphology of M. aeruginosa}

The size and abundance of colonies submitted to the treatments were compared to the control experiment. Thus, the most potent inducer of colony formation of M. aeruginosa was the N-dodecanoyl-homoserine lactone (F), both in number and size, causing a sizeable ameboid aspect in cells additionally (Figure 4). Lactones C, E (inducers of cellular and colonies proliferation) and G, I (inhibitors of cellular proliferation but colonies inducers) likewise induced the formation of large colonies, although smaller than lactone F. A similar size was also detected in colonies treated with lactones $B$ and $D$, which were inhibitors of proliferation and colony formation. Lactone A practically did not affect morphology. 

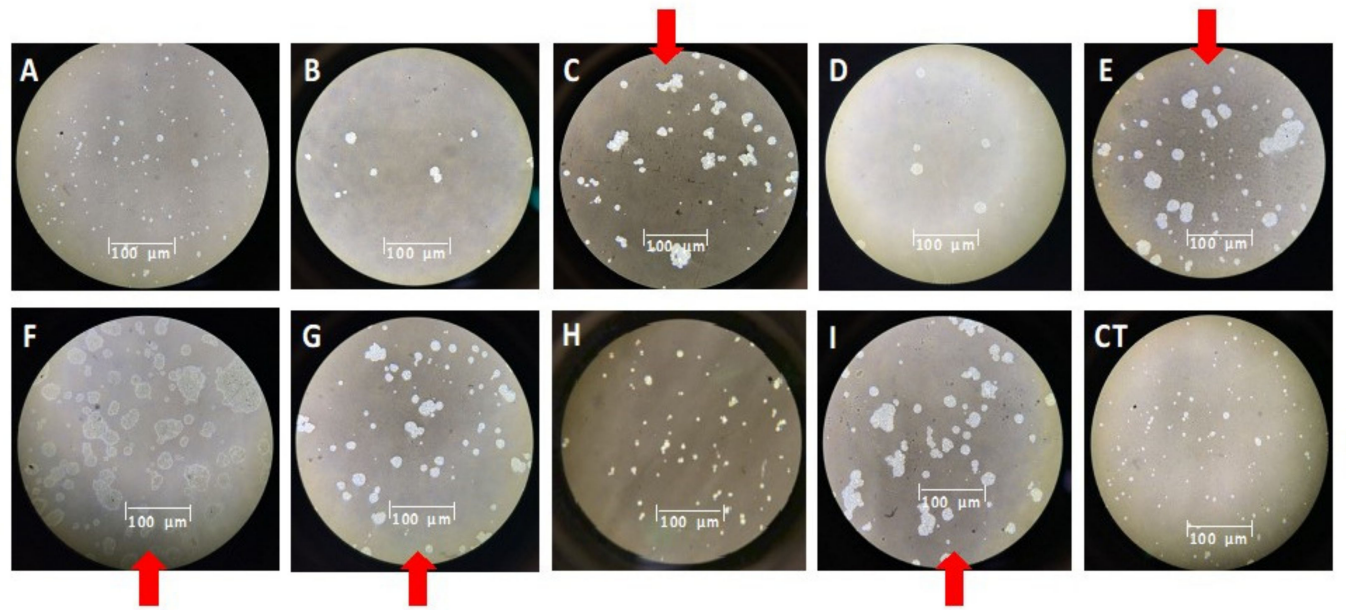

Figure 4. Effect of lactones on colony morphology (10X) of M. aeruginosa. 3-oxodecanoyl (A), N(3-oxododecanoyl)-L-homoserine lactone (B), N-hexanoyl-L-homoserine lactone (C), N-decanoyl (D), heptanoyl (E), N-dodecanoyl-L-homoserine lactone (F), N-octanoyl (G), N-(3-oxooctanoyl)-Lhomoserine lactone $(\mathbf{H})$, butyryl-DL-homoserine lactone $(\mathbf{I})$. The red arrows show the largest colonies. Lactone F induced more colonies of larger size, while lactones C, E, H, G, and I only induced more colonies. A more negligible effect was observed with lactones B and D, but lactone A was virtually inactive.

\subsection{Effect of Vanillin in the Induction of Cell Proliferation and Colony Formation of M. aeruginosa by Lactones}

The ability of vanillin to modify changes caused by lactones in M. aeruginosa cultures is presented in Figure 5. To determine a more accurate effect of compounds on the proliferation of cyanobacteria, in each graph, the results of the induction of cell proliferation of $M$. aeruginosa are discriminated by the number of colonies and number of cells (Figure 5). The addition of N-hexanoyl (C) and N-dodecanoyl-L-homoserine lactone (F) induced cell proliferation in $M$. aeruginosa, which was detected at $96 \mathrm{~h}$, with a percentage of induction higher than $100 \%$ for the lactone C, and 54\% for F, respectively, whereas lactones B and $\mathrm{H}$ did not cause changes. However, the addition of $328.62 \mathrm{nM}$ vanillin to cultures previously treated with $4 \mathrm{nM}$ of lactones caused appreciable effects on cell proliferation (Figure 5). Thus, the induction capacity of cell proliferation of lactones $\mathrm{C}$ and $\mathrm{F}$ was neutralized by vanillin, still below the control using only lactone ( $80 \%$ and $74 \%$ respectively), although lactone B at $96 \mathrm{~h}$ showed a slight reduction effect (25\%). Further, only four lactones, B, C, F, and $\mathrm{H}$ were selected since these compounds showed differential effects in the previously described assays.

To deactivate the effect caused by vanillin, a mixture of this compound and lactone was applied to the cultures of $M$. aeruginosa. Thus, the effect of lactone B and $\mathrm{H}$ was reestablished, but in the case of lactones $C$ and $F$, the capacity to induce cell proliferation activity was not recovered. On the other hand, in all assays, cell proliferation levels after the addition of lactone $\mathrm{H}$ and vanillin remained practically unchanged.

A similar analysis was made in colony formation where the main induction effect was achieved with the application of lactone F (73\%), and a minor effect was caused by lactones $\mathrm{B}$ and $\mathrm{C}$. Again, the addition of vanillin drastically reduced the inducer effect of the lactones B and F (48\% and 75\% respectively) and at a lower level the lactone C. The mixture of lactone and vanillin restored the inducing effect of lactones B and F colonies, but not with lactone $\mathrm{C}$. The mixture of vanillin and lactone $\mathrm{H}$ caused an increase in the number of colonies.

As previously mentioned, it must be considered that these observations were made at $96 \mathrm{~h}$, while the results shown initially (Figure 2) were made at $72 \mathrm{~h}$. 


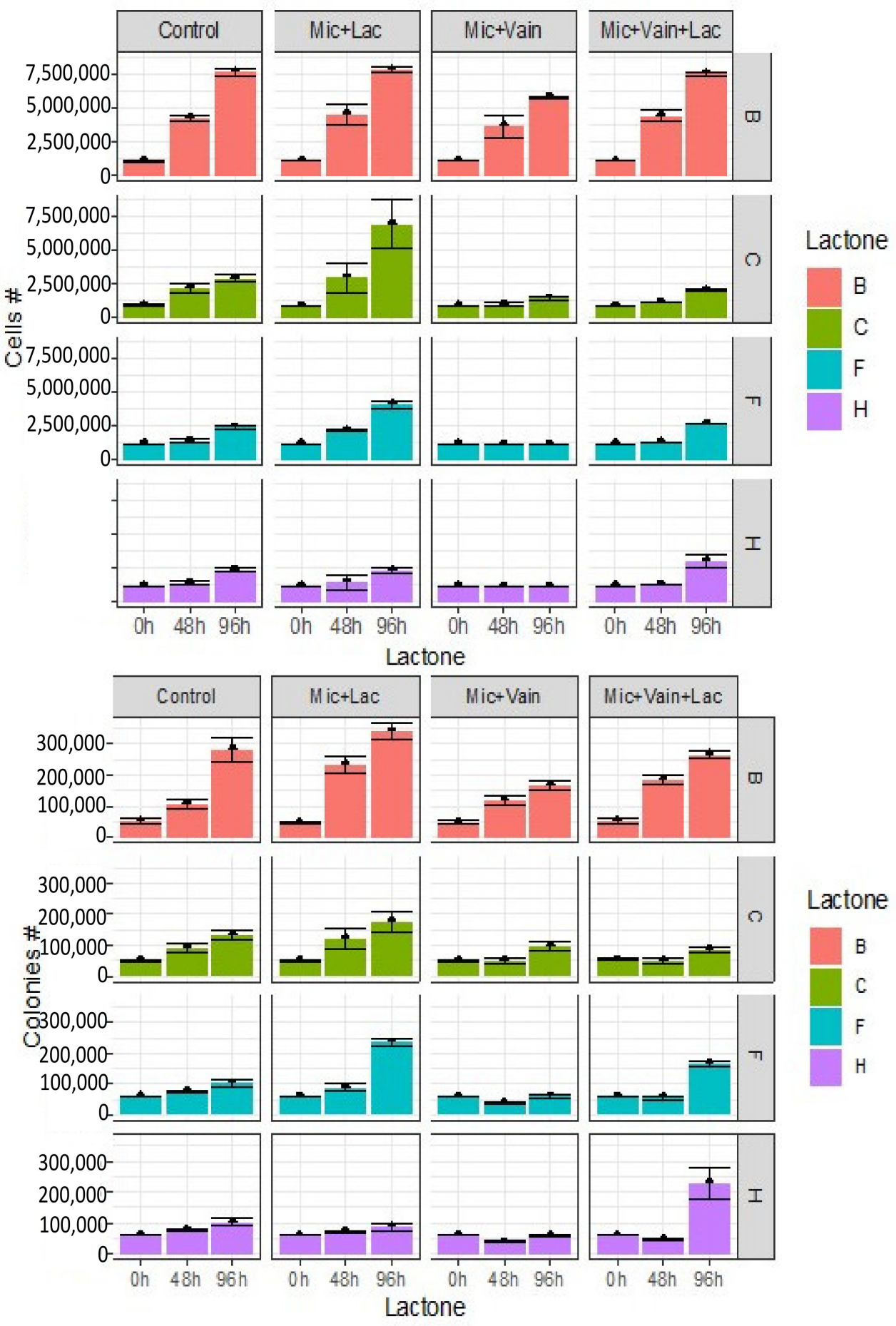

Figure 5. Effect of vanillin on cell proliferation (above) and colony formation (below) of the lactones N-(3-oxododecanoyl) (B), N-hexanoyl (C), N-dodecanoyl (F), and N-(3-oxodecanoyl)-L-homoserine (H), together with the inhibitor (vanillin). Mic: M. aeruginosa, Vai: vanillin, Lac: Lactone. Overall, vanillin was an inhibitor of cell proliferation and colony formation. Its effect even irreversibly blocks the inducing effect of lactones $\mathrm{C}$ and $\mathrm{F}$.

The appearance of colonies of $M$. aeruginosa undergoing treatment with three lactones and vanillin is displayed in Figure 6. Thus, N-(3-oxo-octanoyl)-L-homoserine lactone H causes a deformation of the mucilage, which will likely lead to its decomposition. In the case of n-dodecanoyl lactone F, addition did not seem to reverse vanillin's inhibitory effect. 


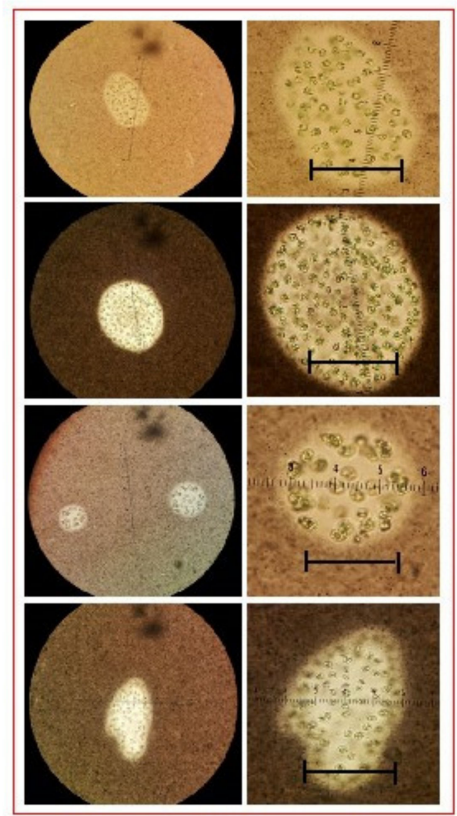

$\mathrm{N}$-Hexanoyl-L homoserine lactone (C)

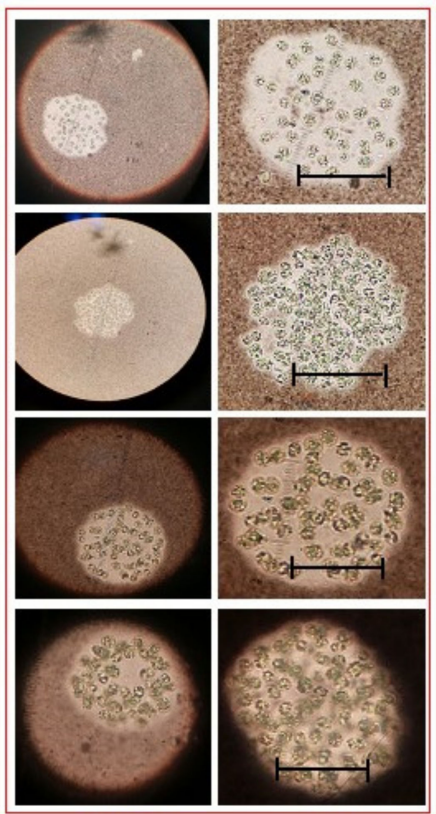

N-(3-Oxooctanoyl)-Lhomoserine lactone $(H)$
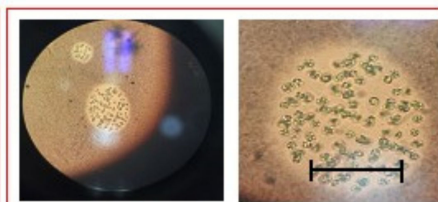

Control
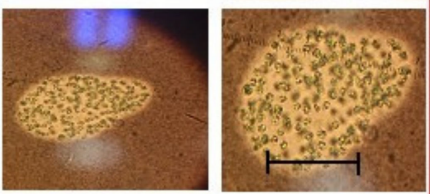

Mic+Lac
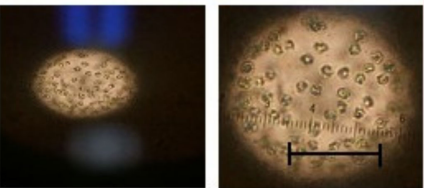

Mic+Vai
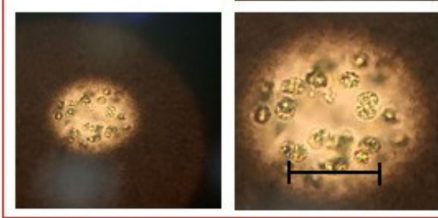

Mic+Vai+Lac

N-Dodecanoyl-L-homoserine lactone (F)

Figure 6. The appearance of M. aeruginosa colonies exposed to substances N- hexanoyl (C), N-dodecanoyl (F), and N-3oxooctanoyl-L homoserine lactone (H). 10X and 40X. Scale bar: $25 \mu \mathrm{m}$. Mic + Lac: M. aeruginosa with lactone; Mic + Vai: M. aeruginosa treated with vanillin. Mic + Lac: Mic + Vai + Lac M. aeruginosa treated with lactone and then vanillin.

\subsection{Effect of Lactones on Microcystin Toxins Production in M. aeruginosa}

This assay allowed us to detect the microcystins LA, RR, and YR as a total because there is cross-reactivity with ELISA. Three controls were used for this purpose; the first one was a standard culture, and the others used microcystin mixtures at 0.2 (CP1) and 0.6 (CP2) $\mathrm{ppb}$ as standards (Figure 7). Besides, a kit calibrator (CN), without microcystins was also used. The largest inducer of microcystin synthesis was N-(3-oxododecanoyl)-L-homoserine lactone (B) with a concentration of $0.73 \mathrm{ppb}$, so, near to $300 \%$ of the increase was detected. Additionally, N-hexanoyl-homoserine lactone (C) similarly induced high levels (more than $100 \%$ ) of these compounds at $0.56 \mathrm{ppb}$. The $\mathrm{F}$ and $\mathrm{H}$ lactones were inducers of the synthesis of toxins, at levels of $98 \%$ and $85 \%$, and slightly, the lactone $\mathrm{E}(56 \%)$.

The other lactones A, D, G, and I, were practically inactive, and the values obtained were not significant. According to the Tukey test, they were in the same group as the negative control.

\subsection{Induction of Cell Proliferation of Cylindrospermopsis raciborskii}

Cylindrospermum raciborskii was studied to analyze whether the observed effect in Microcystis aeruginosa also occurred in other cyanobacteria. This cyanobacteria is a producer of saxitoxin, a potent neurotoxin [14].

The biological profile of C. raciborskii was different from that of M. aeruginosa and could be classified into two types. The first corresponds to cell proliferation inducers, such as $\mathrm{N}$-octanoyl (G), increasing by $20 \%$ (Figure 8 ). The second group included all other lactones and these were considered medium to strong cellular proliferation inhibitors, especially lactones $\mathrm{A}$ and $\mathrm{E}$. 


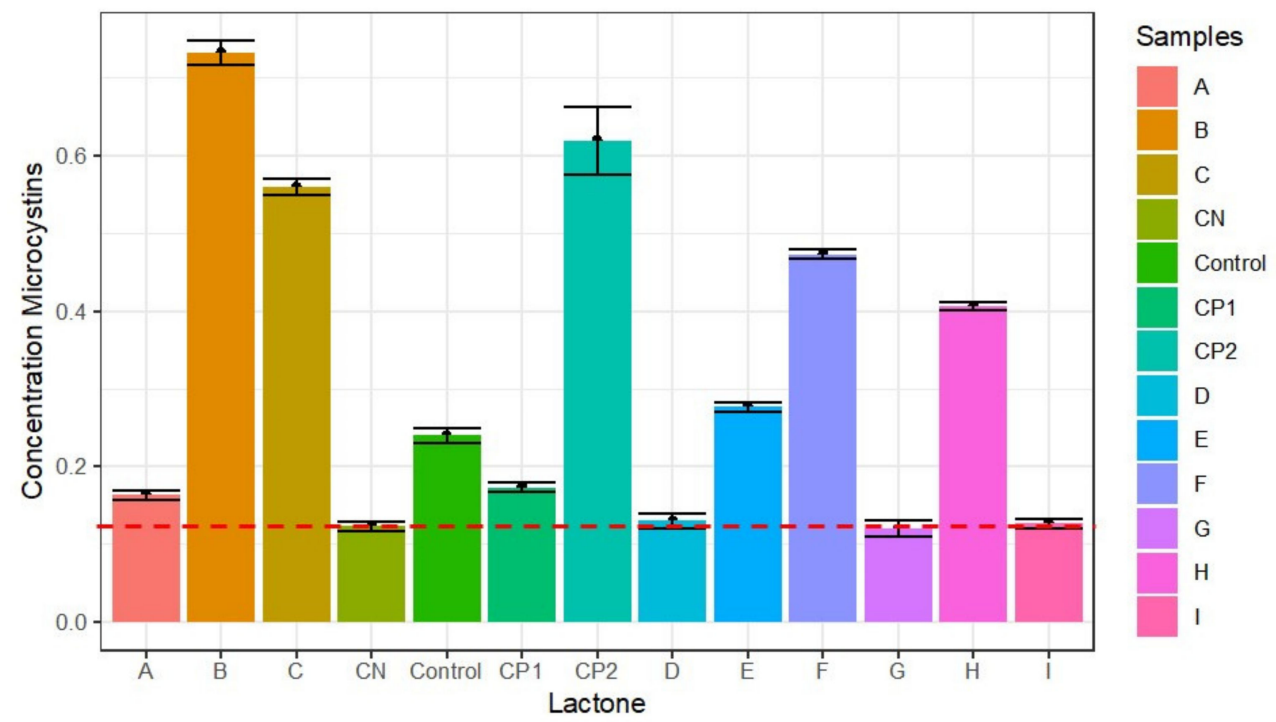

Figure 7. Effects of nine lactones on the production of microcystins in M. aeruginosa, detected by ELISA. CP1 and CP2 are the controls with 0.2 and $0.6 \mathrm{ppb}$ of microcystins, N-(3-oxodecanoyl) (A), N-(3-oxododecanoyl (B), N-hexanoyl (C), N-decanoyl (D), N-heptanoyl (E), N-dodecanoyl (F), Noctanoyl (G), N-(3-oxooctanoyl (H), N-butyryl-homoserine lactone (I). CN: kit calibrator, without microcystins. Lactones $\mathrm{B}$ and $\mathrm{C}$ were potent inducers of microcystins, but lactones $\mathrm{F}$ and $\mathrm{H}$ were also good inducers. The others were inactive. The detection of microcystins in control could be caused by a false positive since the reagent detects microcystins LA, RR, and YR.

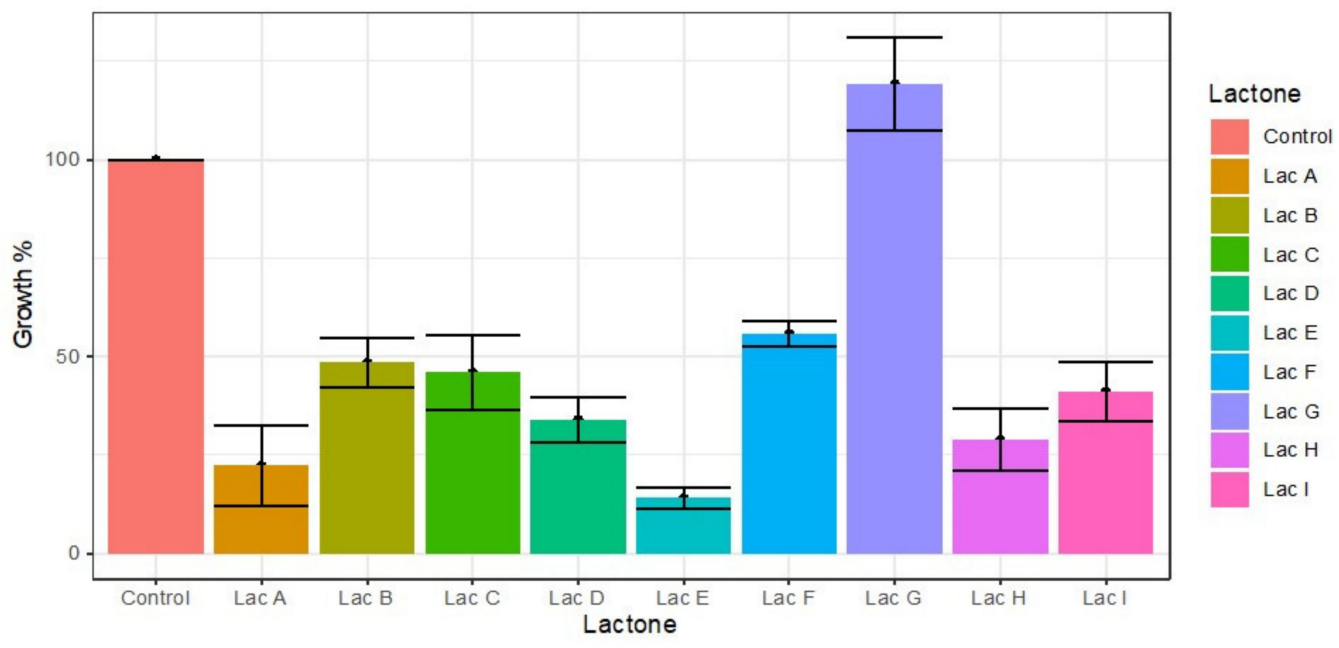

Figure 8. Effect of lactones on the cell proliferation of C. raciborskii: 3-oxodecanoyl (A), N(3-oxododecanoyl)-L-homoserine lactone (B), N-hexanoyl (C), decanoyl (D), heptanoyl (E), Ndodecanoyl (F), N-octanoyl (G), N-(3- oxo-octanoyl (H), and N-butyryl-homoserine lactone (I). Except for lactone $\mathrm{G}$, which had a slight inducing effect, the substances analyzed exhibited inhibitory effects on C. raciborskii cell proliferation, mainly lactones A and E.

\subsection{Effect of Lactones on Saxitoxin Production of Cylindrospermopsis raciborskii}

Practically all lactones caused increases in the levels of saxitoxin (Figure 9). So, 3-oxodecanoyl-L-homoserine lactone (A), N-(3-oxododecanoyl) (B), heptanoyl (E), and $\mathrm{N}$-octanoyl $(\mathrm{G})$ were potent inducers and displayed increases in saxitoxin concentration of $450 \%, 330 \% 320 \%$, and $275 \%$, respectively. A moderate effect was also displayed by the lactones N-hexanoyl (C) (222\%), N-dodecanoyl (F) (213\%), and N-butyryl (I) (195\%). On the other hand, lactones decanoyl (D) and N-(3- oxo-octanoyl $(\mathrm{H})$ only provoked a slight inducer effect. 


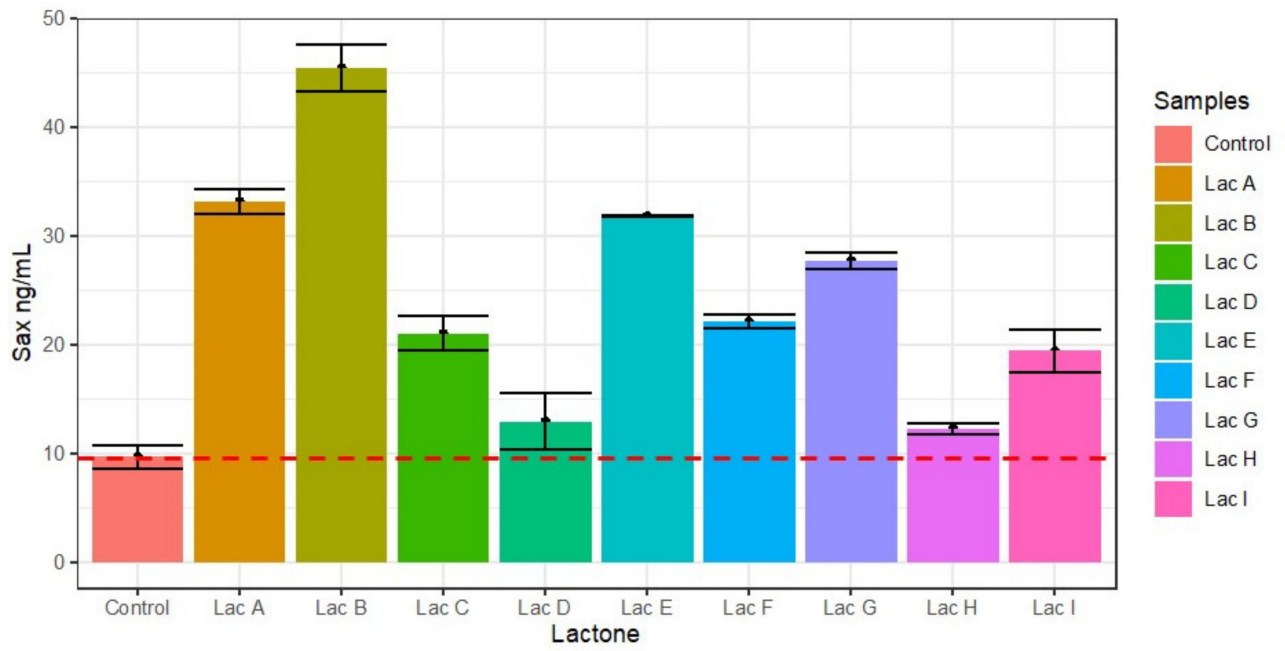

Figure 9. Effect of lactones on the production of saxitoxin. 3-oxodecanoyl (A), N-(3-oxododecanoyl)L-homoserine lactone (B), N-hexanoyl (C), decanoyl (D), heptanoyl (E)), N-dodecanoyl (F), Noctanoyl (G), N-(3- oxo-octanoyl (H), and N-butyryl-homoserine lactone (I). Lactones A, B, E, and G induced saxitoxin synthesis above $275 \%$ to almost $450 \%$ to the control, while lactones $\mathrm{D}$ and $\mathrm{H}$ were virtually inactive.

To establish the existence of quorum sensing in cyanobacteria, we have carried out a biochemical study to determine the effects of several homoserine lactones in M. aeruginosa and C. raciborskii cultures. Table 1 shows the effects of lactones assayed on the elicitation of several processes involving quorum sensing in M. aeruginosa and C. raciborskii.

Table 1. General effects of several lactones in Microcystis aeruginosa and Cylindrospermopsis raciborskii.

\begin{tabular}{|c|c|c|c|c|c|c|c|c|c|c|c|}
\hline \multirow{4}{*}{ Lactone/\# Carbon Atoms } & \multicolumn{9}{|c|}{ M. aeruginosa } & \multirow{3}{*}{\multicolumn{2}{|c|}{$\begin{array}{c}\text { C. raciborskii } \\
\text { Metabolic } \\
\text { Effect }\end{array}$}} \\
\hline & \multirow{2}{*}{\multicolumn{3}{|c|}{ Metabolic Effect }} & \multicolumn{6}{|c|}{ Effect of Vanillin } & & \\
\hline & & & & \multicolumn{3}{|c|}{ Cell Proliferation } & \multicolumn{3}{|c|}{ Colonies Formation } & & \\
\hline & $\begin{array}{l}\text { Cell } \\
\text { Prol } \\
\end{array}$ & $\begin{array}{c}\text { Col } \\
\text { Form }\end{array}$ & Microc & Lact $^{a}$ & $\operatorname{Van}^{b}$ & $\begin{array}{l}\text { Lact + } \\
\text { Van }^{c}\end{array}$ & $\operatorname{Lac}^{a}$ & $\operatorname{Van}^{b}$ & $\begin{array}{r}\text { Lact } \\
\text { Van }^{c} \\
\end{array}$ & $\begin{array}{l}\text { Cell } \\
\text { Prol } \\
\end{array}$ & Saxit \\
\hline Butiryl (I), C4 & - & ++++ & - & NA & NA & NA & NA & NA & $\mathbf{N A}$ & -- & ++ \\
\hline Hexanoyl (C), C6 & ++ & ++++ & +++ & ++++ & WE & WE & + & - & - & -- & ++ \\
\hline Heptanoyl (E), C7 & ++++ & ++++ & + & NA & NA & NA & NA & NA & NA & 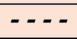 & +++ \\
\hline Octanoyl (G), C8 & - & ++++ & - & NA & NA & $\mathbf{N A}$ & NA & NA & NA & ++ & +++ \\
\hline N-(3-Oxooctanoyl) (H), C10 & +++ & ++++ & ++ & WE & - & WE & WE & WE & +++ & --- & + \\
\hline Decanoyl (D), C10 & -- & WE & - & NA & NA & NA & NA & NA & NA & -- & + \\
\hline N-(3-Oxodecanoyl (A), C10 & - & WE & -- & NA & NA & NA & NA & NA & NA & $\cdots$ & +++ \\
\hline N-Dodecanoyl (F), C12 & ++++ & ++++ & +++ & ++ & WE & + & +++ & -- & - & -- & ++ \\
\hline N-(3-Oxododecanoyl) (B), C12 & -- & WE & ++++ & WE & - & WE & + & 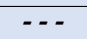 & - & -- & ++++ \\
\hline
\end{tabular}

Lact: Lactone; Van: vanillin; Cell Prol: Cell Proliferation; Col For: Colonies Formation; Microc: Microcystins production; Saxit: Saxitoxin. NA: Not analyzed. ++++ Strong proliferative effect, + Reduced proliferative effect; - - - - Strong inhibitory effect, - Reduced proliferative effect, WE: Without effect. ${ }^{a}$ Respect to the control, ${ }^{b}$ Respect to the lactone, ${ }^{\mathrm{c}}$ Respect to vanillin.

These compounds displayed several chain lengths attached to the lactone ring and occasionally an additional carbonyl group. Lactones I and $\mathrm{C}$ were shorter (C4 and C6), E, $\mathrm{G}$, and $\mathrm{H}$ medium size length (C7, and C8 in the last two), and A, B, D, F longer (C10-C12). Two of them, I and D, were assayed like isomeric mixtures.

Thus, in M. aeruginosa, the main effect is detected in the potent induction of colony formation with almost all lactones, although three of them, A, B, and D, did not respond to this stimulus. As for cell proliferation, several lactones were inhibitors, specifically I, G, $\mathrm{D}$, and B. Regarding the synthesis of toxins, lactone C, F, and B were potent inducers of microcystins synthesis to a lesser extent $\mathrm{H}$ and $\mathrm{E}$. However, cell morphology effects such as those shown in Figure 6 could modify toxins' concentration, possibly due to cell lysis. 
The effects of vanillin, a recognized Quorum Sensing inhibitor, were only determined with lactones $\mathrm{C}, \mathrm{B}, \mathrm{H}$, and $\mathrm{F}$ in $M$. aeruginosa. However, there was a somewhat different performance from the first assay because the observation times changed, and in the case of vanillin, they were analyzed at $96 \mathrm{~h}$ of growth. Thus, lactones $\mathrm{C}$ and F increased cell proliferation, but the strongest was the first. Vanillin addition neutralizes the inducing effect, especially in the case of lactone $\mathrm{C}$. Only lactone $\mathrm{F}$ had an appreciable inducing effect on the colony formation, although vanillin's addition neutralized and caused a decrease in the effect of all lactones, especially B and F.

A mixture of lactone and vanillin was simultaneously administered to the cultures to determine whether vanillin is a competitive inhibitor of lactone activity. There was an almost total recovery with lactones $B$ and $F$ on cell proliferation, but not with lactone $C$, indicating an irreversible effect. Similar effects were noticed on colony formation, except for the mixture with lactone $\mathrm{H}$, a colonies inducer. This result could be explained by differences in the concentration of vanillin and lactone. It is important to analyze the molecular effect of vanillin since it seems to cause an irreversible inhibition in two critical events in the formation of blooms. Unfortunately, its role in the synthesis of toxins, nor C. raciborskii cultures, was not assessed.

Also, there was a clear difference between $M$. aeruginosa and C. raciborskii cultures since there was a dominant inducing effect of cell proliferation, especially in forming colonies with most lactones in the first one. C. raciborskii are species that forms dispersive blooms that do not accumulate on the water's surface like other cyanobacteria but are distributed throughout the depth of the water body. So, in C. raciborskii, the effects were exclusively on the cell proliferation, and the only active proliferation being lactone G; everyone else was potent inhibitors, especially lactones A and E. So, it may be that the mechanism of this process will be mediated by another class of molecules other than acylhomoserine-lactones.

There does not appear to be a clear relationship between structure and modulating activity in both cyanobacteria, as molecules of short, long, or media chains do not have a pattern with respect to any of the activities analyzed. However, hexanoyl-homoserinelactone $\mathrm{C}$ (C6-AHL), oxo-octanoyl-homoserine-lactone (H) (3-oxo-C8-AHL), dodecanoylhomoserinelactone F (C12-AHL), and 3-oxo-dodecanoyl- homoserinelactone B (3-oxoC12-AHL) significantly affected all parameters studied in terms of cell proliferation, colony formation, and microcystins synthesis. In C. raciborskii, it was found that only homoserinelactone $\mathrm{C}$ (C6-AHL) affected cell proliferation and saxitoxin synthesis.

\section{Discussion}

The production of cyanobacterial blooms has become a health problem because of the chronic effects of the toxins generated. This seems to be a worsening issue due to global warming and the massive emission of chemicals into the sea and other water sources [15-17]. Due to several factors, such as large volumes of water, the extension of reservoirs, and the risks to human health and the environment of algicide compounds, chemical methods for the control of cyanobacteria are not feasible. On the other hand, physical methods require a large amount of infrastructure and reduce operational capacity. However, understanding the biochemical processes involved in bloom formation could help design prevention and even control methods.

The role of quorum sensing in cyanobacteria has been discussed, and there is no conclusive evidence so far. Therefore, a relatively recent article entitled 'Does Microcystis aeruginosa have Quorum Sensing?' [18], determining autoinducer lactones' presence is proposed as proof of this. In a 30-day culture of M. aeruginosa, the authors detected $18.0 \mathrm{nM}$ of a compound by HPLC from $1.03 \times 10^{7}$ cells $/ \mathrm{mL}$. Through two bioreporters, Agrobacterium sp. and Chromobacterium sp., the presence of an unusual lactone, not reported before, was confirmed. As definitive proof, they induced biofilms in new cultures of $M$. aeruginosa with extracts from old cultures. In our case, the presence of the nine lactones studied as inductors could not be detected by HPLC/MS, which in the first instance could be attributed to the growing time of eight days, while in the work of Zhai et al. [18], the 
cultures were a month old. The duration of eight days was selected because, after nine days, the cultures suffer a marked deterioration, with rapid and progressive browning of the biomass, indicating chemical and physical decomposition.

Other researchers have detected octanoyl-homoserine lactone (C8-AHL) by HPLC/MS in axenic cultures of the cyanobacteria Gloeothece sp [19]. Moreover, the response to acylhomoserine lactones has been reported in Trichodesmium sp. Similarly, when cultures of these cyanobacteria were treated with these lactones, they doubled their activity to assimilate phosphate from dissolved organic phosphorus, favoring quorum sensing processes [20]. This result indicates that autoinducer lactones are effective promoters of cyanobacteria's growth and development processes, not the other way around. However, Romero et al. [21] found an inhibitory effect of nitrogen fixation in Anabaena sp. with lactones of different sizes. Similarly, in the Swiss lakes, auto-inducing lactones were detected in cyanobacteria assemblies of Planktothrix rubescens and Microcystis wesenbergii [22], although the producer organism remains doubtful because it worked with water from the lake, which could have other lactone-synthesizing bacteria.

Not only the synthesis of the lactones themselves of a specific type of cyanobacteria could trigger a bloom, since in the aquatic environment there are also other bacteria and cyanobacteria associated with this effect. For this reason, the stress in any of them could induce the synthesis of lactones, which would affect the other sensitive species in the same area. It has already been established, for example, that there could be a synergistic relationship between some cyanobacteria and green algae, whose secretome indicates the mediation of quorum sensing molecules [23]. Additionally, through metagenomics, it has been found that marine dinoflagellates and microbial communities are established metabolic relationships mediated by quorum sensing, and even the opposite inhibitory phenomenon, called inhibitor quorum quenching [24].

Biochemical responses to the application of exogenous lactones undoubtedly indicate the existence of quorum sensing in M. aeruginosa and C. raciborskii for the following reasons:

- In this regard, it has been said that $C$. raciborskii induces the formation of colonies in $M$. aeruginosa and inhibits their growth [25], confirming cyanobacterial communication. Experiments are currently underway to determine the production of lactones $M$. aeruginosa and C. raciborskii.

- There was a specific and selective response. Thus, each lactone appears to be involved in a quorum sensing process. This fact could explain why a mixture of lactones is produced. Similarly, there could be selectivity within the same cyanobacteria, as the effects found were different in M. aeruginosa and C. raciborskii.

Quorum sensing helps explain the formation and evolution of cyanobacterial blooms perfectly, whereby features such as temperature, radiation, $\mathrm{pH}$, or nutrient levels could cause the primary stress. Therefore, the synthesis of self-inducing lactones is induced, accelerating cell proliferation, biofilm formation, and toxin synthesis.

Although we have previously reported that some molecules inhibit the growth and production of the toxin microcystin LR, this does not appear to be a feasible solution [11], as massive amounts of chemicals are required to cover a single dam, potentially leading to severe organoleptic and toxicological effects. For this reason, a microbiological and biochemical surveillance system would be more appropriate to detect endogenous and exogenous bloom inducers by controlling microbial populations associated with cyanobacteria, in addition to the dumping of wastes from human and industrial activity.

\section{Conclusions}

In short, for both types of cyanobacteria, M. aeruginosa and C. raciborskii, there appears to be a general communication mechanism mediated by autoinductive lactones, but these compounds act during different events within cyanobacterial development and in a specific way. Moreover, the addition of lactone $F$ to cultures causes profound modifications in the synthesis of several molecules in M. aeruginosa cultures. Furthermore, it is feasible that other substances produced by human activity, such as agrochemicals, human and 
industrial waste, and poultry and livestock farms, could be responsible for triggering blooms, mummifying the inducing effect of natural lactones.

Author Contributions: Conceptualization, methodology, N.H. and F.E.; investigation and funding acquisition, N.H; writing-review and editing, N.H. and F.E. All authors have read and agreed to the published version of the manuscript.

Funding: This research was funded by MINCIENCIAS (Colombia), grant number FP44842-049-2016.

Data Availability Statement: The data presented in this study will be made available on request from the corresponding author.

Acknowledgments: Authors thank to Ricardo Echenique (Facultad de Ciencias Naturales y Museo, Universidad Nacional de La Plata, Argentina) and Cristina Giraldo (Universidad de Antioquia) for analytical assistance. To Aloysio da S. Ferrão-Filho (Instituto Oswaldo Cruz (Fiocruz, Brazil) for technical and scientific assistance.

Conflicts of Interest: The authors declare no conflict of interest.

\section{References}

1. Huisman, J.; Codd, G.A.; Paerl, H.W.; Ibelings, B.W.; Verspagen, J.M.H.; Visser, P.M. Cyanobacterial blooms. Nat. Rev. Microbiol. 2018, 16, 471-483. [CrossRef] [PubMed]

2. Wang, J.; Ding, L.; Li, K.; Huang, H.; Hu, H.; Geng, J.; Xu, K.; Ren, H. Estimation of spatial distribution of quorum sensing signaling in sequencing batch biofilm reactor (SBBR) biofilms. Sci. Total Environ. 2018, 612, 405-414. [CrossRef] [PubMed]

3. Chorus, I.; Welker, M. Toxic Cyanobacteria in Water, 2nd ed.; CRC Press: Boca Raton, FL, USA, 2021.

4. Buratti, F.M.; Manganelli, M.; Vichi, S.; Stefanelli, V.; Scardala, S.; Testai, E.; Funari, E. Cyanotoxins: Producing organisms, occurrence, toxicity, mechanism of action and human health toxicological risk evaluation. Arch. Toxicol. 2017, 91, 1049-1130. [CrossRef] [PubMed]

5. Loise, S.; Calado, D.M.; Souza, G.; Pires, T.; Leite, B.; Cristina, H.; Assis, S. Depuration time and sublethal effects of microcystins in a freshwater fi sh from water supply reservoir. Chemosphere 2018, 210, 805-815. [CrossRef]

6. Gurbuz, F.; Uzunmehmetoğlu, O.Y.; Diler, Ö.; Metcalf, J.S.; Codd, G.A. Occurrence of microcystins in water, bloom, sediment and fish from a public water supply. Sci. Total Environ. 2016, 562, 860-868. [CrossRef] [PubMed]

7. Lejars, M.; Hajnsdorf, E. Antisense RNA world in bacteria. Biochim. Biophys. Acta 2020, 1863, 194489. [CrossRef] [PubMed]

8. Anderson, R.A. Algal Culturing Techniques; Elsevier/Academic Press: Amsterdam, The Netherlands, 2005.

9. Hoffmann, L.; Komárek, J.; Kaštovský, J. System of cyanoprokaryote (cyanobacteria)—State in 2004. Algol. Stud. 2005, 117, 95-115. [CrossRef]

10. Papenfort, K.; Bassler, B. Quorum-Sensing Signal-Response Systems in Gram-Negative Bacteria. Nat. Rev. Microbiol. 2016, 14, 576-588. [CrossRef]

11. Herrera, N.; Florez, M.T.; Velasquez, J.P.; Echeverri, F. Effect of Phenyl-Acyl Compounds on the Growth, Morphology, and Toxin Production of Microcystis aeruginosa Kützing. Water 2019, 11, 236. [CrossRef]

12. Dos S Vieira, J.M.; de P Azevedo, M.T.; de Oliveira Azevedo, S.M.F.; Honda, R.Y.; Corrêa, B. Microcystin production by Radiocystis fernandoi (Chroococcales, Cyanobacteria) isolated from a drinking water reservoir in the city of Belém, PA, Brazilian Amazonia region. Toxicon 2003, 42, 709-713. [CrossRef] [PubMed]

13. R Core Team. 2020. Available online: http://www.r-project.org/index.html (accessed on 31 January 2021).

14. Osti, J.A.S.; Podduturi, R.; Schlüter, L.; Liu, T.; Moraes, M.D.E.B.; Jorgensen, N.O.G. Monitoring of saxitoxin production in lakes in Denmark by molecular, chromatographic and microscopic approaches. Harmful Algae 2021, 101, 101966. [CrossRef]

15. Hofer, U. Climate change boosts cyanobacteria. Nat. Rev. Microbiol. 2018, 16, 122-123. [CrossRef] [PubMed]

16. Paerl, H.W.; Paul, V.J. Climate change: Links to global expansion of harmful cyanobacteria. Water Res. 2012, 46, 1349-1363. [CrossRef] [PubMed]

17. Moe, S.J.; Haande, S.; Couture, R.-M. Climate change, cyanobacteria blooms and ecological status of lakes: A Bayesian network approach. Ecol. Model. 2016, 337, 330-347. [CrossRef]

18. Zhai, C.; Zhang, P.; Shen, F.; Zhou, C.; Liu, C. Does Microcystis aeruginosa have quorum sensing? FEMS Microbiol. Lett. 2012, 336, 38-44. [CrossRef] [PubMed]

19. Sharif, D.I.; Gallon, J.; Smith, C.J.; Dudley, E. Quorum sensing in Cyanobacteria: N -octanoyl-homoserine lactone release and response, by the epilithic colonial cyanobacterium Gloeothece PCC6909. ISME J. 2008, 2, 1171-1182. [CrossRef]

20. Van Mooy, B.A.S.; Hmelo, L.R.; Sofen, L.E.; Campagna, S.R.; May, A.L.; Dyhrman, S.T.; Heithoff, A.; Webb, E.A.; Momper, L.; Mincer, T.J. Quorum sensing control of phosphorus acquisition in Trichodesmium consortia. ISME J. 2011, 6, 422-429. [CrossRef] [PubMed]

21. Romero, M.; Muro-pastor, A.M.; Otero, A. Quorum sensing N-acylhomoserine lactone signals affect nitrogen fixation in the cyanobacterium Anabaena sp. PCC7120. FEMS Microbiol. Lett. 2011, 315, 101-108. [CrossRef] 
22. Braun, E.; Bachofen, R. Homoserine-lactones and microcystin in cyanobacterial assemblages in Swiss lakes. Hydrobiologia 2004, 522, 280. [CrossRef]

23. Gautam, K.; Tripathi, J.K.; Pareek, A.; Sharma, D.K. Growth and secretome analysis of possible synergistic interaction between green algae and cyanobacteria. J. Biosci. Bioeng. 2019, 127, 213-221. [CrossRef] [PubMed]

24. Huang, X.; Zhu, J.; Cai, Z.; Lao, Y.; Jin, H.; Yu, K. Profiles of quorum sensing (QS)-related sequences in phycospheric microorganisms during a marine dinoflagellate bloom, as determined by a metagenomic approach. Microbiol. Res. 2018, 217, 1-13. [CrossRef] [PubMed]

25. Mello, M.M.E.; Soares, M.C.S.; Roland, F.; Lürling, M. Growth inhibition and colony formation in the cyanobacterium Microcystis aeruginosa induced by the cyanobacterium Cylindrospermopsis raciborskii. J. Plankton Res. 2012, 34, 987-994. [CrossRef] 